\title{
CBCT-based evaluation of integrity of cortical sinus close to periapical lesions
}

\section{Oséas SANTOS JUNIOR Lucas Rodrigues PINHEIRO Otávio Shoiti UMETSUBO Marcelo Gusmão Paraíso CAVALCANTI}

Universidade de São Paulo - USP, School of Dentistry, Department of Stomatology, São Paulo, SP, Brazil
Declaration of Interests: The authors certify that they have no commercial or associative interest that represents a conflict of interest in connection with the manuscript.

Corresponding Author:

Marcelo Cavalcanti

E-mail:mgpcaval@usp.br

DOI: 10.1590/1807-3107BOR-2015.vol29.0019

Submitted: Feb 21, 2014

Accepted for publication: Sep 08, 2014

Lat revision: Nov 27, 2014
Abstract: The purpose of the present study was to test the applicability of cone beam computed tomography $(\mathrm{CBCT})$ to evaluate the integrity of the cortical sinus close to periapical lesions. Two observers analyzed samples of 64 alveoli of first molar roots in macerated swine maxillas prepared with perchloric acid to simulate periapical lesions. The specimens were evaluated using CBCT (55-mm high and 100-mm diameter cylinder at 0.2-mm voxel resolution) for the presence of oroantral communication (OAC) caused by the simulated lesions. Sensitivity, specificity, and accuracy were calculated. Fair values were obtained for accuracy (66\%-78\%) and good values for specificity $(70 \%-98 \%)$, whereas the values for sensibility showed relevant variation $(41 \%-78 \%)$. For this reason, the interobserver agreement score was weak. CBCT proved capable of evaluating the integrity of the cortical sinus (absence of oroantral communication) when it lies close to an apical periodontitis lesion. However, the low interobserver agreement reflects the difficulty in performing diagnoses when OAC is adjacent to a periapical lesion, using the acquisition protocol adopted in this research. This could be attributed to the high level of image noise.

Keywords: Periapical Periodontitis; Models, Animal; Cone-Beam Computed Tomography; Diagnosis; Maxillary Sinus.

\section{Introduction}

Early diagnosis of odontogenic sinusitis is important, because it is a common condition, and differs in pathophysiology and management from other sinus diseases of different etiology. Odontogenic sinusitis occurs when the sinus membrane is perforated, possibly caused by periapical lesions in maxillary teeth. ${ }^{1}$

Several studies have demonstrated the importance of performing a diagnosis when the source of sinusitis is odontogenic, so that an effective treatment plan can be established for the adequate healing of tooth infection, mainly by endodontic treatment, and for the effective prevention of sinusitis recurrence and evolution. ${ }^{1,2,3}$

When CBCT imaging is available, these image data may be used in preventing post-treatment complications, in the diagnosis of pathology and in improved visualization of the anatomical structure in the posterior maxilla. Apical periodontitis accessed with conventional images is frequently underestimated; however, the application of a higher radiation dose cautions use of CBCT except when justified or supported by clinical examination. ${ }^{4}$ Anatomical and pathological maxillary sinus involvement in posterior upper 
teeth is considerably high, thus giving $\mathrm{CBCT}$ a role of clinical relevance in performing radioanatomical and radiodiagnostic observations of the maxillary sinus floor and the alveolar bone and teeth. ${ }^{5}$

The proximity between the root apices of the maxillary teeth and the maxillary sinus may generate an image of overlapping structures in two-dimensional (2D) radiographs, hiding a periapical lesion that compromises the integrity of the cortical sinus and triggers odontogenic sinusitis. ${ }^{3,6,7,8}$ Several studies have shown better sensitivity and specificity of CT, as compared with $2 \mathrm{D}$ radiographs, but there is a lack of studies comparing CBCT with the gold standard. ${ }^{9}$

For this reason, the objective of the present study was to test CBCT applicability in evaluating the integrity of the cortical sinus close to periapical lesions. The sensitivity, specificity, accuracy and reproducibility of $\mathrm{CBCT}$ were tested in evaluating simulated periapical lesions in order to detect the presence or absence of oroantral communication (OAC) in macerated swine maxillas, thereby establishing this set of determinants (simulated periapical lesions/ oroantral communication/ macerated swine maxillas) as the gold standard.

\section{Methodology Preparation of samples}

Swine maxillas $(n=20)$ were macerated by boiling them in water, as the preferred method to preserve bone quality of the sample. ${ }^{10}$ They were then scanned by CBCT to verify the integrity of the alveolar bone of first molar mesiobuccal and distobuccal roots. Maxillas with alveolar bone defects or third molars near the region of interest (ROI) were excluded from the sample. The eligible maxillas were selected for lesion simulation $(n=16)$. First molars were extracted after odontosection $(n=32)$, taking care to maintain the integrity of the alveoli and roots, as verified by visual inspection. ${ }^{11}$

The same operator, not involved in interpreting the images, performed erosions in all the alveoli, to simulate lesions. This was done by applying 70\% perchloric acid (Merck Chemicals, Darmstadt, Germany) in the mesiobuccal and distobuccal root alveoli (Figure 1), thus generating the sites $(n=64)$ to be analyzed on CT images. ${ }^{12,13}$

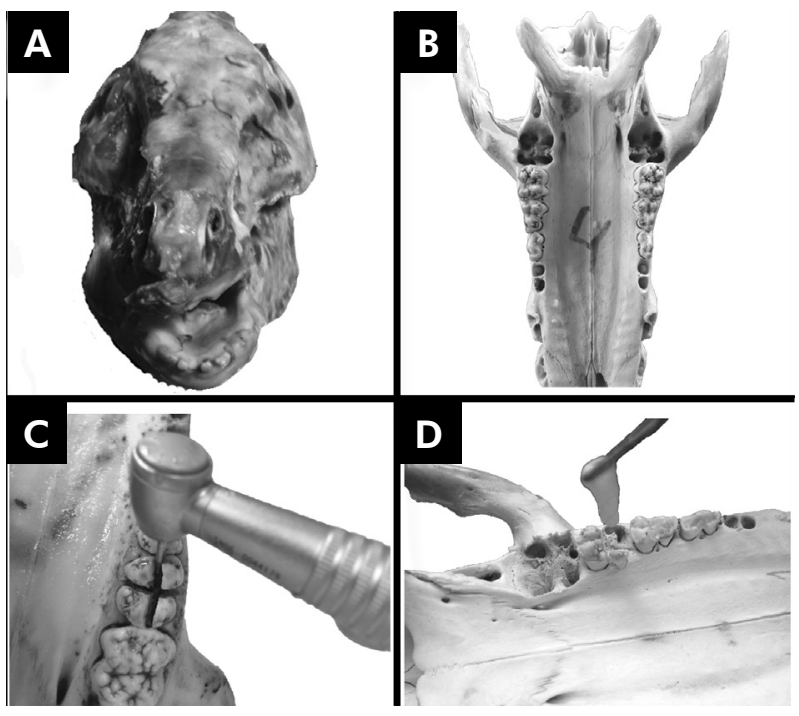

Figure 1. Preparation of samples: A) Pig's head; B) Macerated swine maxilla; C) Odontosection; D) Application of perchloric acid.

Some sites $(\mathrm{n}=32)$ were exposed to acid for a shorter time, to generate incipient lesions (Time 1: 3 hours), whereas others $(n=32)$ were exposed for a longer time, to simulate larger lesions (Time 2: 4 hours). ${ }^{11,14}$ The action of the acid was stopped by rinsing the alveoli in running water after the specified time. The impairment of cortical air spaces evolved in some cases to create the intended disruption, thus generating OAC $(n=22)$. Proof that there was communication between the alveoli and the air space was verified by visual inspection, i.e., by observing a periodontal probe that was introduced into the alveoli, and that passed through the oral cavity into the air space. This is how the gold standard was established for this study. Sites with OAC were recorded, for comparison with diagnosis by CT scans.

\section{Image acquisition}

A water-filled plastic container was used to simulate soft tissue attenuation, such as an in vivo situation. ${ }^{15,16}$ A CBCT (ProMax 3D Max, Planmeca Oy / Helsinki, Finland) scan was performed for each maxilla, with the following acquisition protocol: the limits of the imaging area consisted of a $55-\mathrm{mm}$ high and $100-\mathrm{mm}$ diameter cylinder; the voxel size was $0.2 \mathrm{~mm}$. These parameters were established according to the selection of a default 
acquisition protocol for the maxillary region, made available by this CBCT unit.

Sixteen CBCT scans of maxillas (with periapical lesions simulated in the distobuccal and mesiobuccal roots of first molars on both sides) were obtained using this procedure. There were two double-blinded observers, who were experienced oral and maxillofacial radiologists and also trained and calibrated on the tomographic features of periapical lesions affecting the cortical sinus, with and without OAC. Each observer analyzed 64 sites in the CBCT scans.

After image acquisition, the data were stored in Digital Imaging Communication in Medicine (DICOM) file format and imported by OsiriX version 3.8.1 (OsiriX Foundation, Geneva, Switzerland), an open-source DICOM viewer installed in a iMAC 27 - Mac OS X Snow Leopard workstation (Apple, Cupertino, USA).

\section{Radiographic assessment}

The entire volume of each sample was analyzed by the observers, using multiplanar reconstructed axial, coronal (Figure 2), and sagittal (Figure 3) images. The sequence of observations was randomized by the Random.org website (www.random.org).

The interpretation time was not restricted, and the same images were analyzed repeatedly after a 2-week interval, so that the observers would not remember the previous image interpretation. Presence of OAC was diagnosed by a dichotomous (yes/no) evaluation, and the scores were labeled as follows: correct identification or absence of OAC

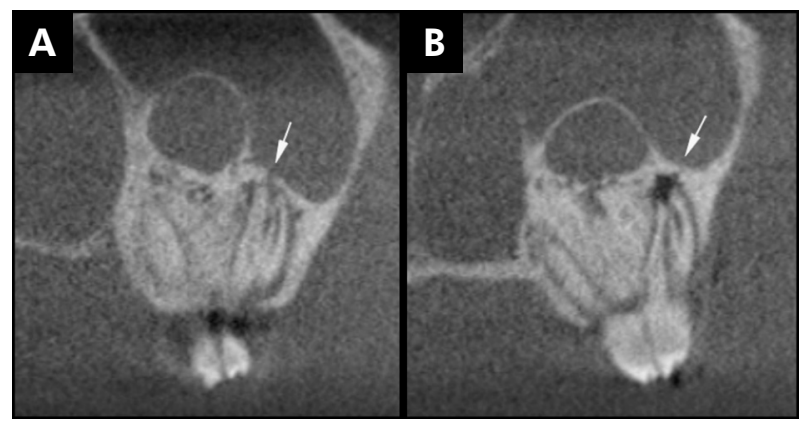

Figure 2. Coronal CBCT images: A) Sample with oroantral communication (arrow) caused by periapical lesion; B) Sample without disruption of cortical sinus (arrow) close to periapical lesion.

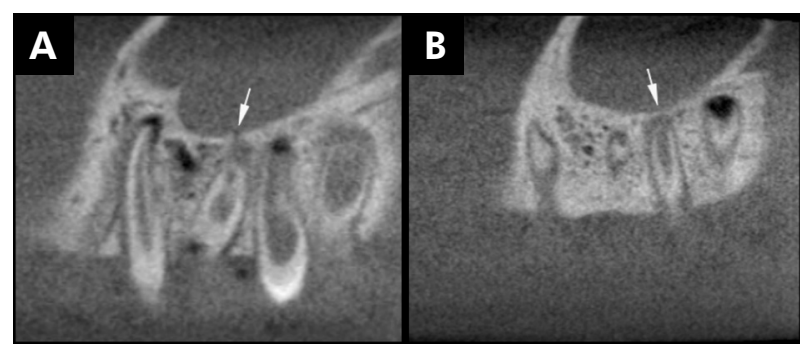

Figure 3. Sagittal CBCT images: A) Sample with oroantral communication (arrow) caused by periapical lesion; B) Sample without disruption of cortical sinus (arrow) close to periapical lesion.

(true negative - TN); correct identification of OAC (true positive - TP); identification of OAC in a maxillary sinus floor without disruption (false positive - FP); and failure to identify OAC in a maxillary sinus floor with disruption (false negative - FN).

\section{Data analysis}

Statistical analyses were carried out using the Validity and Kappa tests. The k coefficients were calculated to assess the degree of intra- and interobserver agreement, and were scored as weak (0.20-0.39), moderate (0.40-0.59), and relevant (0.60-0.79). ${ }^{17}$ These coefficients made it possible to check the tomographic diagnosis against the gold standard. The level of significance was set at $\mathrm{a}=0.05$. The data were analyzed using SPSS software (v 17.0.0; SPSS Inc.; Chicago, USA).

Sensitivity, specificity and accuracy of the analyses were calculated as follows: Sensitivity $=\mathrm{TP} /(\mathrm{TP}+\mathrm{FN})$, Specificity $=\mathrm{TN} /(\mathrm{TN}+\mathrm{FP})$, Accuracy $=(\mathrm{TP}+\mathrm{TN}) /$ $(\mathrm{TP}+\mathrm{FP}+\mathrm{FN}+\mathrm{TN})$. The positive and negative predictive values (PPV and NPV) were also calculated to determine the probability of true diagnosis. Statistical analyses were performed using BioEstat software (v 5.3; Bioestat, Belém, Brazil).

\section{Results}

The whole sample $(\mathrm{n}=64)$ was submitted to perchloric acid: one part $(n=32)$ was exposed at Time 1, resulting in 7 sites with OAC, and the other part $(n=32)$ was exposed at Time 2 , resulting in 15 communications between the alveoli and the maxillary sinus, yielding 64 sites with periapical lesions and 22 OACs. 
Table 1 presents the kappa values for interobserver agreement in detecting OAC caused by periapical lesions, and Table 2 presents the kappa values for intraobserver agreement. The intraobserver values ( 0.49 to 0.56 ) were considered as moderate levels of agreement, and the interobserver values ( 0.21 to 0.35 ), as low levels of agreement.

Table 3 shows the overall TP and FP, as well as TN and $\mathrm{FN}$ results for the diagnosis of periapical lesions that damage cortical sinus integrity. The results for overall sensitivity, positive predictive values, specificity, negative predictive values and accuracy are shown in Table 4. These data represent all the observations for both observers. The values for sensitivity and specificity varied for each observer. Accuracy rates were roughly similar among the observers, and significant values $(p<.05)$ were obtained ( $66 \%$ to $78 \%)$.

Table 1. Interobserver reliability.

\begin{tabular}{lcc}
\hline \multirow{2}{*}{ Value } & First evaluation & Second evaluation \\
\cline { 2 - 3 } & Observer $1 \times$ Observer 2 & Observer $1 \times$ Observer 2 \\
\hline $\mathrm{k}$ & 0.3469 & 0.2120 \\
$\mathrm{p}$ & 0.00001 & 0.0373 \\
\hline
\end{tabular}

Table 2. Intraobserver reliability.

\begin{tabular}{lcc}
\hline Value & $\begin{array}{c}\text { First evaluation } x \\
\text { Second evaluation }\end{array}$ & $\begin{array}{c}\text { First evaluation } x \text { Second } \\
\text { evaluation }\end{array}$ \\
\cline { 2 - 3 } & Observer 1 & Observer 2 \\
\hline $\mathrm{k}$ & 0.4928 & 0.5573 \\
$\mathrm{p}$ & $<0.00001$ & $<0.00001$ \\
\hline
\end{tabular}

Table 3. Percentage of True (T) and False (F) diagnosis of oroantral communication caused by periapical lesions.

\begin{tabular}{lcccccc}
\hline & \multicolumn{2}{c}{ Observer 1 } & \multicolumn{2}{c}{ Observer 2 } & \multicolumn{2}{c}{ Total } \\
\cline { 2 - 7 } & $\mathrm{T}(\%)$ & $\mathrm{F}(\%)$ & $\mathrm{T}(\%)$ & $\mathrm{F}(\%)$ & $\mathrm{T}(\%)$ & $\mathrm{F}(\%)$ \\
\hline Fist Evaluation & 78 & 22 & 73 & 27 & 76 & 24 \\
Second Evaluation & 77 & 23 & 66 & 34 & 71 & 29 \\
\hline
\end{tabular}

\section{Discussion}

The true etiology of maxillary sinusitis plays an important role in clinical management, mainly because treatment of odontogenic sinusitis is different from that of rhinogenic sinusitis. Sinusitis diagnosis is
Table 4. Sensitivity, positive predictive values, specificity, negative predictive values and accuracy rates (\%) calculated according to diagnosis of oroantral communication caused by periapical lesions.

\begin{tabular}{lcccc}
\hline & \multicolumn{2}{c}{ First evaluation } & \multicolumn{2}{c}{ Second evaluation } \\
\cline { 2 - 5 } & Observer 1 & Observer 2 & Observer 1 & Observer 2 \\
\hline Sensitivity (\%) & 41 & 78 & 55 & 60 \\
PPV (\%) & 90 & 60 & 70.59 & 50 \\
Specificity (\%) & 98 & 71 & 88 & 70 \\
NPV (\%) & 75.93 & 85.29 & 78.72 & 76.32 \\
Accuracy (\%) & 78 & 74 & 77 & 66 \\
\hline
\end{tabular}

based mostly on clinical signs, although radiological findings may be very valuable in identifying etiological factors. ${ }^{5}$ Many studies assess etiological factors, like periodontal disease, carious cavities, large restorations, surgical procedures, apical periodontitis and endodontic therapy in the posterior maxilla, as well as mucosal thickening in the maxillary sinus. ${ }^{1,3,4}$

A shared consensus in these studies is that the closer proximity of posterior tooth roots with the maxillary sinus floor increases the risk of odontogenic sinusopathy, mucositis and sinusitis. Nevertheless, studies reveal that infection and inflammatory mediators can affect the sinus mucosa without perforating the cortical bone of the sinus floor. ${ }^{18}$ In this study, we simulated lesions in order to induce OAC as a relevant cause of bacterial colonization of odontogenic origin, thus triggering complications.

The proximity between the root apices of the maxillary teeth and the maxillary sinus can generate an image of overlapping structures in 2D radiographic examination, hiding incipient lesions and damage to the integrity of a cortical sinus possibly having OAC. ${ }^{3,6,7,8}$ Thus, this in vitro study used $70 \%$ perchloric acid to mimic periapical lesions (occurring from inflammatory reactions, and therefore having more irregular boundaries than those made by drills). This procedure made simulated initial lesions look like those observed in vivo, thereby enabling simulation of incipient lesions, which are more difficult to detect. ${ }^{11,12,13,19}$

The anatomical proximity between the root apex and the cortical air space is more relevant than the size of a periapical lesion in establishing OAC. ${ }^{3,8}$ Therefore, the longer acid etching time was not a 
determining factor for establishing OAC. Nonetheless, the analysis of this anatomic relationship (maxillary sinus and apex) was performed by CBCT with good reliability. This corroborates previous studies ${ }^{5}$ and shows the clinical relevance of the radioanatomical observation allowed by CBCT. It may also be one of the reasons for the high specificity and accuracy of the results.

Pigs are considered a good species to represent human beings in studying craniofacial bones, because their anatomy, morphology, and bone density in this region is similar to that of humans. This makes swine maxillary bones a good sample for radiological studies. ${ }^{20,21}$ Moreover, the current research corroborated that porcine maxillas are considered a good model for studying this anatomical region. However, some difficulties were found in standardizing the positioning of the ROI of the sample in the center of the FOV during the tomographic acquisitions, due to maxilla size variation. This is precisely one of the limitations observed in this study. That is to say, the use of human skulls would be more suitable for similar studies in CBCT devices with pre-standardized acquisition protocols.

Several studies have compared and attested to higher sensitivity and specificity of CBCT scans, as compared with 2D X-rays to diagnose periapical pathologies in maxillary teeth. ${ }^{4,5,22,23}$ A systematic review on radiological diagnosis reported that CBCT has not been compared with a gold standard. ${ }^{9}$ For this reason, the current research tests a possible comparison between CBCT images and a gold standard (simulated lesions in the maxillas generating or failing to generate OAC, attested by visual inspection and periodontal probe).

The size of the FOV and the size of the reconstructed voxels are parameters that may vary in different imaging protocols of the same CBCT unit, and that may influence image quality. A large FOV with a small voxel reconstruction generates images with a relevant level of noise. ${ }^{24,25}$ The CBCT unit used in this study allows the option of choosing pre-defined imaging protocols. Accordingly, we opted for the protocol related to the region of the maxillary teeth, especially because of the difficulty in standardizing the positioning of the ROI (apical region of the first molar adjacent to the cortical sinus) of the porcine model for image acquisition. This protocol generated images with a relatively large FOV and small voxels. As a result, we found image noise, reported by the observers as the main difficulty in performing their analyses. Another possible reason for the lower image quality could be attributed to the water-filled container, used in the present study, in which the samples were totally immersed when the CBCT scan was performed. ${ }^{16,26}$ The water-bearing container was intended to cause beam attenuation, and leveled the values of our in vitro study to clinic levels. Because the CBCT unit used in this study did not allow free manipulation of the parameters, filters and calibration could have caused the images to have a higher level of noise. This limitation affected our in vitro research, thus hindering the detection of small OACs adjacent to the periapical lesion.

The results showed high CBCT specificity (70\%-98\%) in the evaluation of the integrity of the cortical sinus close to the apical periodontitis lesion, attributed to the good assessment of the relationship between the maxillary sinus and the posterior root apices. However, the sensitivity values were lower (41\%-78\%), possibly attributed to the difficulty in analyzing the existence of OAC adjacent to the periapical lesion, caused by image noise. The choice of an acquisition protocol with a smaller FOV could attenuate the level of image noise. ${ }^{15,16}$ Therefore, we believe that the choice of an acquisition protocol with a smaller FOV and the positioning of the ROI at the center of the FOV can improve these rates.

Although the accuracy indices were similar among the observers, the sensitivity values were distinct. This resulted in a low level of interobserver agreement. The most obvious difficulty, and one reported by the observers, was diagnosing a cortical sinus rupture in the presence of an artifact. Clinically, the findings could infer that CBCT is a valuable adjunct to anatomical evaluation of posterior maxilla and diagnosis of periapical lesions in the root apices close to the maxillary sinus floor (all simulated lesions at both times - Time 1 and Time 2 - were easily noticed by observers). However, OAC-related limitation exists in regions where the periapical lesion is 
adjacent to the cortical sinus, leading to inconclusive diagnosis of this condition.

\section{Conclusion}

Our study concluded that CBCT has fair accuracy in evaluating the integrity of the cortical sinus close to a periapical lesion (values ranging from $66 \%$ to $78 \%$ ) and good specificity in detecting the absence of oroantral communication (values ranging from $70 \%$ to $98 \%$ ). However, the low interobserver agreement evidenced the difficulty in diagnosing oroantral communication adjacent to periapical lesions, possibly caused by the high level of image noise. Other acquisition protocols

\section{References}

1. Legert KG, Zimmerman M, Stierna P. Sinusitis of odontogenic origin: pathophysiological implications of early treatment. Acta Otolaryngol. 2004 Aug;124(6):655-63.

2. Cymerman JJ, Cymerman DH, O'Dwyer RS. Evaluation of odontogenic maxillary sinusitis using cone-beam computed tomography: three case reports. J Endod. 2011 Oct;37(10):1465-9.

3. Lee KC, Lee SJ. Clinical features and treatments of odontogenic sinusitis. Yonsei Med J. 2010 Nov;51(6):932-7.

4. Shahbazian M, Vandewoude C, Wyatt J, Jacobs R. Comparative assessment of periapical radiography and $\mathrm{CBCT}$ imaging for radiodiagnostics in the posterior maxilla. Odontology. 2013 Dec 28.

5. Shahbazian M, Vandewoude C, Wyatt J, Jacobs R. Comparative assessment of panoramic radiography and CBCT imaging for radiodiagnostics in the posterior maxilla. Clin Oral Investig. 2014 Jan;18(1):293-300.

6. Oberli K, Bornstein MM, von Arx T. Periapical surgery and the maxillary sinus: radiographic parameters for clinical outcome. Oral Surg Oral Med Oral Pathol Oral Radiol Endod. 2007 Jun;103(6):848-53.

7. Kilic C, Kamburoglu K, Yuksel SP, Ozen T. An assessment of the relationship between the maxillary sinus floor and the maxillary posterior teeth root tips using dental cone-beam computerized tomography. Eur J Dent. 2010 Oct;4(4):462-7.

8. Pagin O, Centurion BS, Rubira-Bullen IR, Alvares Capelozza AL. Maxillary sinus and posterior teeth: accessing close relationship by cone-beam computed tomographic scanning in a Brazilian population. J Endod. 2013 Jun;39(6):748-51.

9. Petersson A, Axelsson S, Davidson T, Frisk F, Hakeberg M, Kvist $\mathrm{T}$, et al. Radiological diagnosis of periapical bone tissue lesions in endodontics: a systematic review. Int Endod J. 2012 Sep;45(9):783-801.

10. Lee EJ, Luedtke JG, Allison JL, Arber CE, Merriwether DA, Steadman DW. The effects of different maceration techniques should be investigated to improve the sensitivity values in detecting oroantral communication very close to periapical lesions.

\section{Acknowledgments}

Conselho Nacional de Desenvolvimento Científico e Tecnológico - CNPq (Marcelo Cavalcanti Research Productivity Scholarship), Brasília, Brazil, grant no. 303847/2009-3 (to M.G.P.C.); Universal Research Project grant no. 472895/2009-5 (to M.G.P.C.); Coordenação de Aperfeiçoamento de Pessoal de Nível Superior - CAPES (PhD scholarship to O.S.U., and Master's scholarship to O.S.J.); and Fundação de amparo à Pesquisa do Estado de São Paulo - FAPESP (PhD scholarship to L.R.P.).

on nuclear DNA amplification using human bone. J Forensic Sci. 2010 Jul;55(4):1032-8.

11. Sogur E, Grondahl HG, Baksi BG, Mert A. Does a combination of two radiographs increase accuracy in detecting acid-induced periapical lesions and does it approach the accuracy of cone-beam computed tomography scanning? J Endod. 2012 Feb;38(2):131-6.

12. Ozen T, Kamburoglu K, Cebeci AR, Yuksel SP, Paksoy CS. Interpretation of chemically created periapical lesions using 2 different dental cone-beam computerized tomography units, an intraoral digital sensor, and conventional film. Oral Surg Oral Med Oral Pathol Oral Radiol Endod. 2009 Mar;107(3):426-32.

13. Umetsubo OS, Gaia BF, Costa FF, Cavalcanti MG. Detection of simulated incipient furcation involvement by CBCT: an in vitro study using pig mandibles. Braz Oral Res. 2012 Jul-Aug;26(4):341-7.

14. Tsai P, Torabinejad M, Rice D, Azevedo B. Accuracy of conebeam computed tomography and periapical radiography in detecting small periapical lesions. J Endod. 2012 Jul;38(7):965-70.

15. Costa FF, Gaia BF, Umetsubo OS, Cavalcanti MG. Detection of horizontal root fracture with small-volume cone-beam computed tomography in the presence and absence of intracanal metallic post. J Endod. 2011 Oct;37(10):1456-9.

16. Costa FF, Gaia BF, Umetsubo OS, Pinheiro LR, Tortamano IP, Cavalcanti MG. Use of large-volume cone-beam computed tomography in identification and localization of horizontal root fracture in the presence and absence of intracanal metallic post. J Endod. 2012 Jun;38(6):856-9.

17. Landis JR, Koch GG. The measurement of observer agreement for categorical data. Biometrics. 1977 Mar;33(1):159-74.

18. Maillet M, Bowles WR, McClanahan SL, John MT, Ahmad M. Cone-beam computed tomography evaluation of maxillary sinusitis. J Endod. 2011 Jun;37(6):753-7. 
19. Tirrell BC, Miles DA, Brown CE Jr, Legan JJ. Interpretation of chemically created lesions using direct digital imaging. J Endod. 1996 Feb;22(2):74-8.

20. Stembirek J, Kyllar M, Putnova I, Stehlik L, Buchtova M. The pig as an experimental model for clinical craniofacial research. Lab Anim. 2012 Oct;46(4):269-79.

21. Wang S, Liu Y, Fang D, Shi S. The miniature pig: a useful large animal model for dental and orofacial research. Oral Dis. 2007 Nov;13(6):530-7.

22. Lofthag-Hansen S, Huumonen S, Gröndahl K, Gröndahl HG. Limited cone-beam CT and intraoral radiography for the diagnosis of periapical pathology. Oral Surg Oral Med Oral Pathol Oral Radiol Endod. 2007 Jan;103(1):114-9.

23. Bernardes RA, Paulo RS, Pereira LO, Duarte MA, OrdinolaZapata R, Azevedo JR. Comparative study of cone beam com- puted tomography and intraoral periapical radiographs in diagnosis of lingual-simulated external root resorptions. Dent Traumatol. 2012 Aug;28(4):268-72.

24. Barrett JF, Keat N. Artifacts in CT: recognition and avoidance. Radiographics. 2004 Nov-Dec;24(6):1679-91.

25. Librizzi ZT, Tadinada AS, Valiyaparambil JV, Lurie AG, Mallya SM. Cone-beam computed tomography to detect erosions of the temporomandibular joint: Effect of field of view and voxel size on diagnostic efficacy and effective dose. Am J Orthod Dentofacial Orthop. 2011 Jul;140(1):e25-30.

26. Perrella A, Lopes PM, Rocha RG, Fenyo-Pereira M, Cavalcanti MG. Influence of dental metallic artifact from multislice CT in the assessment of simulated mandibular lesions. J Appl Oral Sci. 2010 Mar-Apr;18(2):149-54. 